\title{
Synthesis, Structural and Magnetic Characterization of Ni-Doped ZnO Diluted Magnetic Semiconductor
}

\author{
R. Elilarassi", G. Chandrasekaran
}

Department of Physics, School of Physical, Chemical and Applied Sciences, Pondicherry University, Puducherry, 605014, India

\begin{abstract}
In the present work, $\mathrm{Zn}_{1-\mathrm{x}} \mathrm{Ni}_{\mathrm{x}} \mathrm{O}(\mathrm{x}=0.02,0.04,0.06$ and 0.08$)$ nanoparticles have been synthesized using sol-gel auto-combustion method. The structural and magnetic properties of the Ni-doped $\mathrm{ZnO}$ samples annealed at $800^{\circ} \mathrm{C}$ were characterized by Thermogravimetry/Differential thermal analysis (TG/DTA), X-ray diffractometer (XRD), Scanning electron microscope (SEM), FTIR spectrophotometer, Vibrating sample magnetometer (VSM) and Electron paramagnetic resonance (EPR) spectroscopy. Thermal analysis of as-prepared $\mathrm{Zn}_{0.98} \mathrm{Ni}_{0.02} \mathrm{O}$ sample shows that the synthesis process undergoes two stage weight losses before yielding $\mathrm{Zn}_{0.98} \mathrm{Ni}_{0.02} \mathrm{O}$ nanoparticles. Structural analysis using XRD reveals the formation of hexagonal wurtzite structure. SEM micrographs of $\mathrm{Zn}_{0.98} \mathrm{Ni}_{0.02} \mathrm{O}$ show the presence of spherical nanoparticles and the formation of well defined pores in the sample. FTIR study confirms the formation of $\mathrm{ZnO}$ with the stretching vibrational mode around $525 \mathrm{~cm}^{-1}$. VSM measurement of sample $\left(\mathrm{Zn}_{0.96} \mathrm{Ni}_{0.04} \mathrm{O}\right)$ shows the hysteresis loop at room temperature confirms the ferromagnetic property of the sample. EPR spectra of the nickel doped $\mathrm{ZnO}$ samples suggest that the exchange interaction between $\mathrm{Ni}^{2+}$ ions results in the ferromagnetic nature of the samples.
\end{abstract}

Keywords Ni-doped $\mathrm{ZnO}$, auto-combustion, $\mathrm{X}$-ray diffraction, diluted magnetic semiconductor

\section{Introduction}

Diluted magnetic semiconductors (DMS) obtained by introducing a dilute amount of transition metal ions into semiconductors in which the transport and magnetic properties combined in a single substance. DMS have attracted immense interest among the researchers and intensively studied for their potential technological applications such as generating and manipulating spin-polarized currents[1]. DMS with the use of spin carriers in addition to charge carriers, as an added degree of freedom is a promising candidate material in the field of magnetic recording media[2]. In addition, DMS materials have several advantages such as instant-on computer, increased integration density, higher data processing speed, low electrical energy demand and compatibility of their fabrication processes[3]. Recently, investigations on oxide diluted magnetic semiconductors such as transition metal doped $\mathrm{ZnO}, \mathrm{TiO}_{2}, \mathrm{CeO}_{2}, \mathrm{SnO}_{2}$, have triggered great revolution among the researchers for the emerging field of spintronics device applications. This is so because, a DMS based on III-V semiconductors such as GaAs, InAs doped with transition metals shows ferromagnetism only at very low temperatures[4,5]. For an efficient DMS material, its Curie temperature above room temperature is desired. Transition metal doped $\mathrm{ZnO}$ have been

* Corresponding author:

ezhil1984_r@yahoo.co.uk (R. Elilarassi)

Published online at http://journal.sapub.org/materials

Copyright (C) 2012 Scientific \& Academic Publishing. All Rights Reserved under intense investigation since the theoritical prediction of curie temperature to be above room temperature[6].

Many reports on the nickel doped $\mathrm{ZnO}$ diluted magnetic semiconductor with room temperature ferromagnetism in the form of thin films and nanocrystals[7-11]. Despite a number of reports available on nickel doped $\mathrm{ZnO}$, still lot of controversies persists on the existence and origin of room temperature ferromagnetism in this system. In the present work, we have made an attempt to investigate $\mathrm{Zn}_{1-\mathrm{x}} \mathrm{Ni}_{\mathrm{x}} \mathrm{O}(0.02<\mathrm{x}$ $<0.08)$ using the sol-gel auto-combustion method. The present synthesis method has several advantages such as cost effective, lesser synthesis time, requires low temperature for processing and requisite amount of final product. The results of the structural, thermal and magnetic characterization of synthesized Ni-doped nanocrystals are also presented. The powder samples of different concentration of Ni-doped $\mathrm{ZnO}$ have been characterized with regard to their thermal stability (TGA/DTA), phase(s) (XRD and FTIR), microstructure (SEM), and magnetic properties (VSM and EPR).

\section{Experimental Procedure}

Nanocrystalline $\mathrm{Zn}_{1-\mathrm{x}} \mathrm{Ni}_{\mathrm{x}} \mathrm{O}(\mathrm{x}=0.02,0.04,0.06,0.08)$ powder samples were synthesized by a simple and low-temperature auto-combustion method. The stoichiometric amount of the aqueous solution containing the analytical grade metal nitrates (oxidizer) $\mathrm{Zn}\left(\mathrm{NO}_{3}\right)_{2} 6 \mathrm{H}_{2} \mathrm{O}, \mathrm{Ni}\left(\mathrm{NO}_{3}\right)_{2} 6 \mathrm{H}_{2} \mathrm{O}$, and glycine (fuel) $\mathrm{C}_{2} \mathrm{H}_{5} \mathrm{NO}_{2}$ was calculated based on the total oxidizing and reducing valency of oxidizer and the fuel, which 
serve as a numerical coefficient for the stoichiometric balance such that the equivalence ratio is unity, i.e. $\mathrm{O}: \mathrm{F}=1.0[12]$ and the energy released is at a maximum. Fig.1. Shows the schematic diagram of autocombustion method to prepare nickel doped $\mathrm{ZnO}$ nanoparticles.

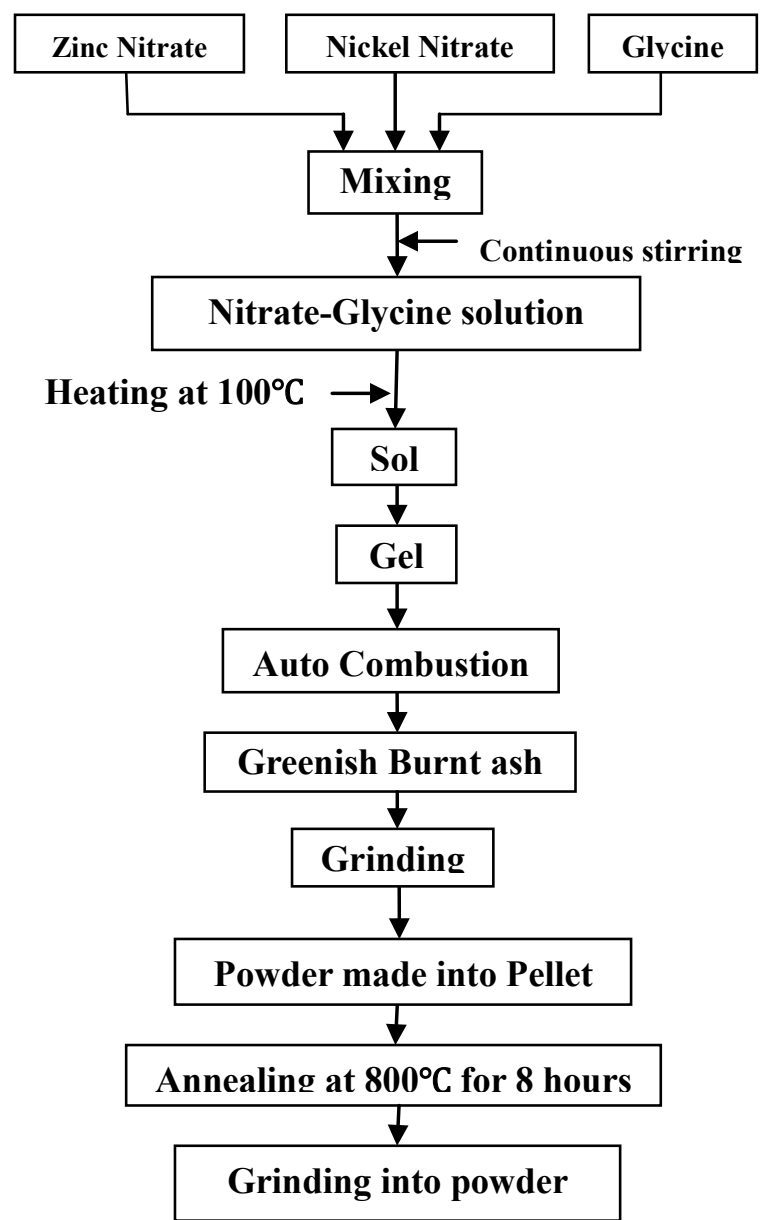

Figure 1. Shows the schematic diagram of autocombustion method to prepare nickel doped $\mathrm{ZnO}$ Nanoparticles

In the present synthesis glycine plays the role of organic fuel which possesses a high heat of combustion, and it offers a platform for the redox reactions to occur between the reactants during the course of combustion. Metal nitrates and glycine were completely dissolved in a $1000 \mathrm{ml}$ beaker to obtain a $100 \mathrm{ml}$ aqueous solution. To mix the precursor solution, the aqueous solution was stirred for about $1 \mathrm{~h}$. The mixed precursor solution was evaporated on a hot plate at $100^{\circ} \mathrm{C}$ under constant stirring and was concentrated by heating until the excess free water was evaporated. Then, the solution converted into a "gel". The "gel" was subsequently swelling into foam and underwent a strong selfpropagating combustion reaction to give a fine powder. The combustion process yields voluminous ash accompanied by dense yellowish fumes. The resulted powders were moss green and became darker with increasing Ni content. The product is gently crushed into a fine powder using the mortar and pestle for an hour and was made as pellets. The pellets were subjected to annealing at $800^{\circ} \mathrm{C}$ for 8 hours and ground for 1 hour for further characterization studies.

\section{Characterization}

Thermogravimetric (TG) and differential thermal analysis (DTA) of the powder sample were carried out at a heating rate of $10^{\circ} \mathrm{C} / \mathrm{min}$ using TA SDT Q600 2960 analyzer (Shimadzu, USA). The crystalline nature and the phase purity of the powder samples were examined by X-ray powder diffraction (XRD) with (PAnalytical Model: X'Pert PRO). The microstructure of the powder sample $\left(\mathrm{Zn}_{0.98} \mathrm{Ni}_{0.02} \mathrm{O}\right)$ was investigated using Scanning Electron Microscope (SEM) (HITACHI Model: S-3400 N). Infrared spectra of the powder samples were recorded using Fourier Transform Infra-Red Spectrophotometer (FTIR) (Nicolet-6700) from 400 to $4000 \mathrm{~cm}^{-1}$ by the $\mathrm{KBr}$ pellet method. Magnetic properties of the $\mathrm{Zn}_{0.96} \mathrm{Ni}_{0.04} \mathrm{O}$ sample were experimentally studied by measuring magnetization as a function of external magnetic field at room temperature using Vibrating sample magnetometer (VSM) (Lakeshore 7404). Electron spin paramagnetic resonance (EPR) characteristics of the powder samples were studied using (JES-TE100 ESR spectrometer). The structural and magnetic measurements of the samples were carried out at room temperature except EPR study performed at $77 \mathrm{~K}$.

\section{Results and Discussion}

\subsection{Thermal Analysis}

TG-DTA analyses of the as-synthesized $\mathrm{Zn}_{0.98} \mathrm{Ni}_{0.02} \mathrm{O}$ powder sample was carried out to determine the appropriate calcination temperature. Fig.2. shows the TG-DTA curves of $\mathrm{Zn}_{0.98} \mathrm{Ni}_{0.02} \mathrm{O}$. The as-synthesized powder $\mathrm{Zn}_{0.98} \mathrm{Ni}_{0.02} \mathrm{O}$ sample was heated in the presence of nitrogen atmosphere at the rate of $10^{\circ} \mathrm{C} / \mathrm{min}$ in the temperature range $115.0^{\circ} \mathrm{C}$ to $180.0^{\circ} \mathrm{C}$ with $8 \%$ weight loss. The weight loss may be due to the evaporation of water content present in the $\mathrm{Zn}-\mathrm{Ni}$ precursors and can be verified from DTA curve that shows an endothermic peak at $172^{\circ} \mathrm{C}$. The second stage of decomposition has been observed in the temperature range of $330^{\circ} \mathrm{C}-405^{\circ} \mathrm{C}$ with an endothermic peak at $380^{\circ} \mathrm{C}$ that indicates a weight loss of $27 \%$ due to the decomposition of $\mathrm{Ni}-\mathrm{Zn}$ precursor to the formation of $\mathrm{Zn}_{0.98} \mathrm{Ni}_{0.02} \mathrm{O}$. Since there is no appreciable change in weight above $400^{\circ} \mathrm{C}$, the calcination temperature of the powder was optimized at $405^{\circ} \mathrm{C}$.

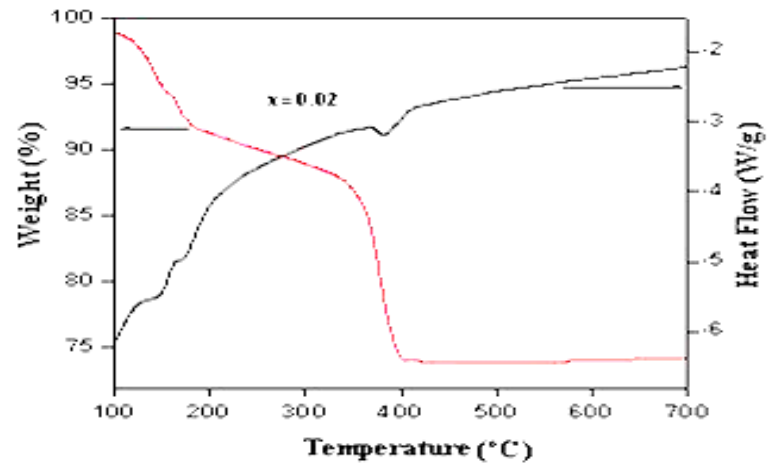

Figure 2. TG/DTA curve of as-prepared $\mathrm{Zn}_{1-\mathrm{x}} \mathrm{Ni}_{\mathrm{x}} \mathrm{O}(\mathrm{x}=0.02)$ sample 


\subsection{Structural Analysis}

Fig. 3 shows the X-ray diffraction patterns of nickel doped $\mathrm{ZnO}$ nanoparticles synthesized using auto-combustion method. The XRD patterns of $\mathrm{Zn}_{1-\mathrm{x}} \mathrm{Ni}_{\mathrm{x}} \mathrm{O}(\mathrm{x}=0.02,0.04$, $0.06,0.08)$ samples annealed at $800^{\circ} \mathrm{C}$ shows the reflection planes indexed to wurtzite hcp structure of $\mathrm{ZnO}$ (space group $P 63 m c$, JCPDF \# 36-1451)[13].

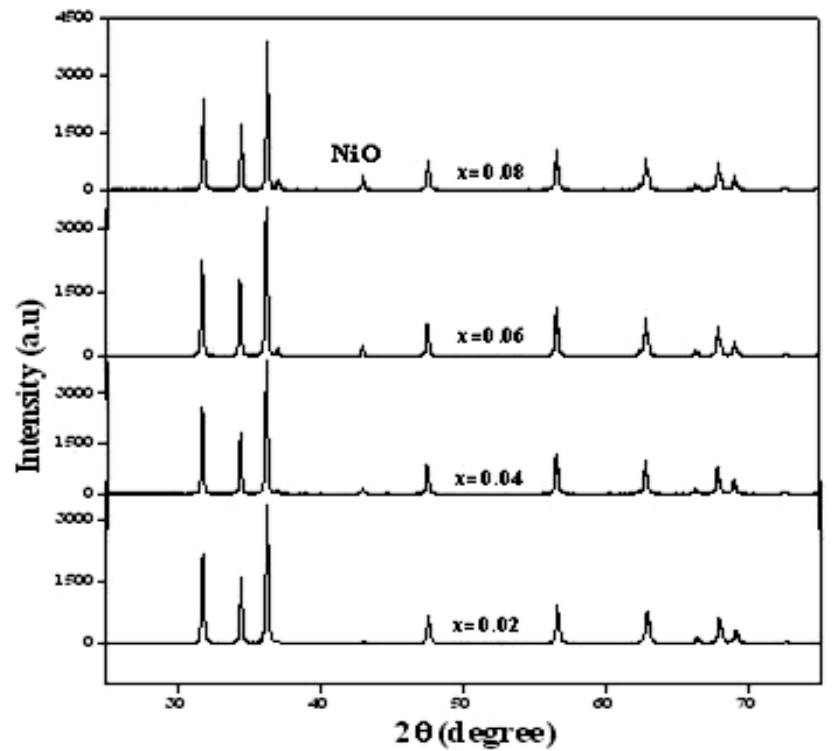

Figure 3. XRD patterns of $\mathrm{Zn}_{1-\mathrm{x}} \mathrm{Ni}_{\mathrm{x}} \mathrm{O}(\mathrm{x}=0.02,0.04,0.06,0.08)$ powders annealed at $800^{\circ} \mathrm{C}$

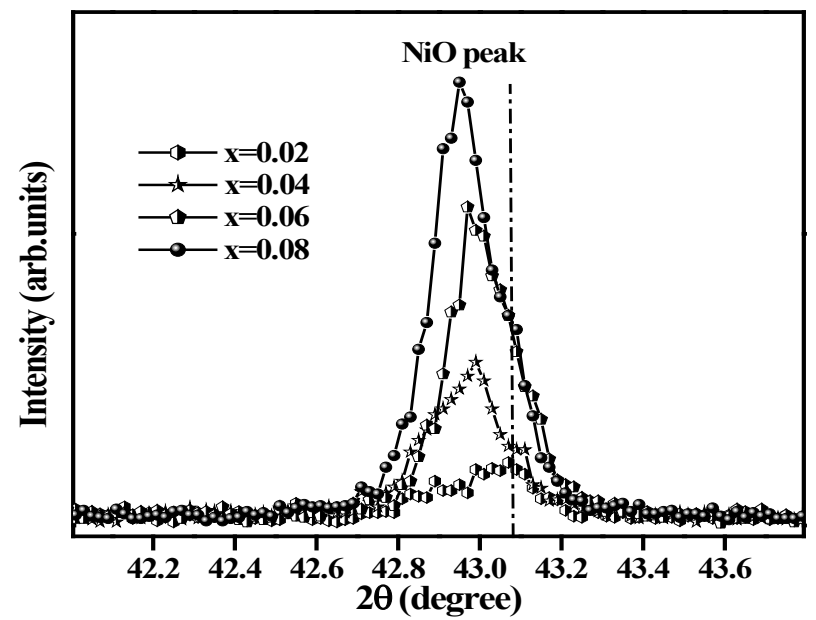

Figure.4. Variation of 2 theta of $\mathrm{NiO}$ peak with the doping concentration of $\mathrm{Zn}_{1-\mathrm{x}} \mathrm{Ni}_{\mathrm{x}} \mathrm{O}$ samples annealed at $800^{\circ} \mathrm{C}$

However, an additional diffraction peak corresponded to $\mathrm{NiO}$ comes into existence at an angle $2 \theta \sim 42.5^{\circ}$, which clearly indicate that phase segregation have occurred in the samples. Fig. 4 shows the variation of 2 theta of $\mathrm{NiO}$ peak with the doping concentration of $\mathrm{Zn}_{1-\mathrm{x}} \mathrm{Ni}_{\mathrm{x}} \mathrm{O}$ samples annealed at $800^{\circ} \mathrm{C}$. It is observed that the intensity of $\mathrm{NiO}$ peak increases sharply and shifts to the lower angle with increasing nickel concentration. The observed left shift in the $\mathrm{NiO}$ diffraction peak from their native place with increasing nickel content is an evident that $\mathrm{NiO}$ is distorted to larger spacing where nickel gets oxidized and incorporated into
$\mathrm{ZnO}$ lattice[14]. Fig. 5. shows the morphology of $\mathrm{Zn}_{0.98} \mathrm{Ni}_{0.02} \mathrm{O}$ powder sample annealed at $800^{\circ} \mathrm{C}$ investigated using SEM. SEM micrographs of the powder sample show the distribution of spherical nanocrystallites with clear boundaries and the formation of well defined pores in the sample.

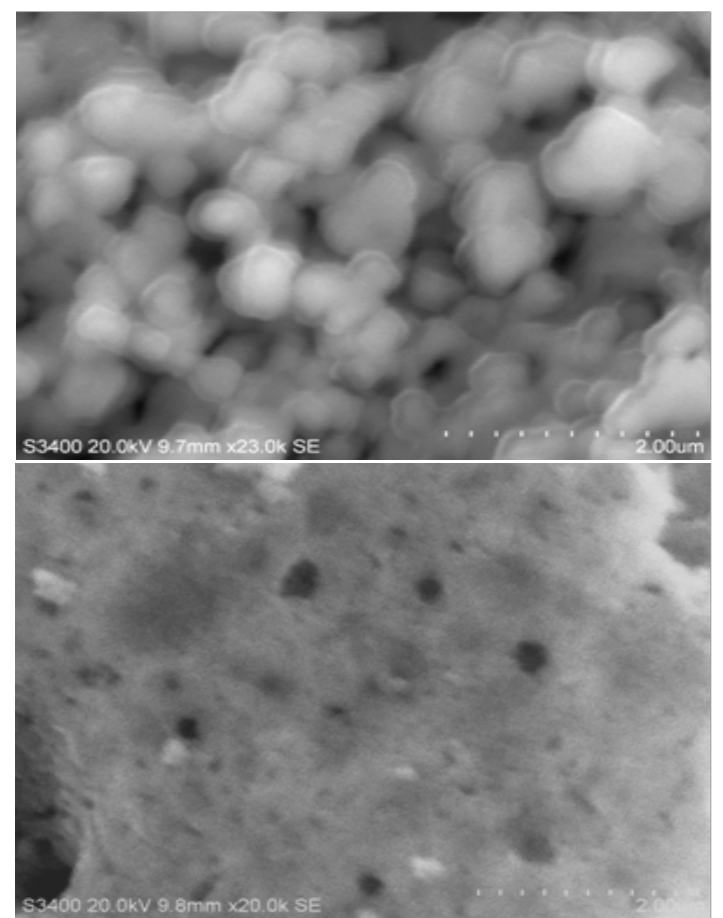

Figure 5. SEM micrographs of $\mathrm{Zn}_{0.98} \mathrm{Ni}_{0.02} \mathrm{O}$ sample annealed at $800^{\circ} \mathrm{C}$

\subsection{FTIR Analysis}

Fig.6. shows the FT-IR spectra of $\mathrm{Zn}_{1-\mathrm{x}} \mathrm{Ni}_{\mathrm{x}} \mathrm{O}$ ( $\mathrm{x}=0.02$, 0.04, $0.06,0.08)$ nanocrystals annealed at $800^{\circ} \mathrm{C}$. From the FTIR spectra, the strong vibrational mode observed at $525 \mathrm{~cm}^{-1}$ is assigned to stretching vibrations of $\mathrm{ZnO}[15]$.

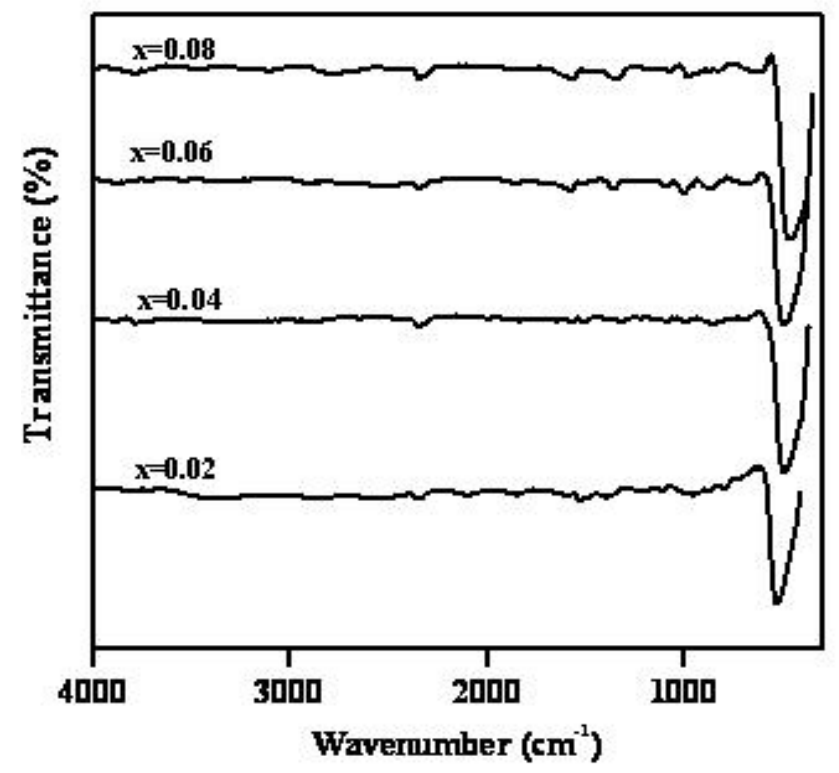

Figure.6. FTIR spectra of $\mathrm{Zn}_{1-\mathrm{x}} \mathrm{Ni}_{\mathrm{x}} \mathrm{O}(\mathrm{x}=0.00,0.02,0.04,0.06,0.08)$ samples annealed at $800^{\circ} \mathrm{C}$ 
The weak absorption bands centred at $1340 \mathrm{~cm}^{-1}$ and 1565 $\mathrm{cm}^{-1}$ attributed to the stretching vibration of $\mathrm{C}=\mathrm{O}$ and $\mathrm{C}=\mathrm{H}$ groups. From the FTIR analysis of the powder samples, it is well understood that all other vibration modes above 600 $\mathrm{cm}^{-1}$ are weak when compared to that of wurtzite $\mathrm{ZnO}$ vibration mode $525 \mathrm{~cm}^{-1}$ clearly depicts the good crystalline quality of the samples.

\subsection{VSM Study}

Magnetization measurements on $\mathrm{Zn}_{0.96} \mathrm{Ni}_{0.04} \mathrm{O}$ nanoparticles annealed at $800^{\circ} \mathrm{C}$ were performed using vibrating sample magnetometer at room temperature. Fig. 7 shows the hysterisis loop obtained from the magnetization $M$ versus field $H$ data for $\mathrm{Zn}_{0.96} \mathrm{Ni}_{0.04} \mathrm{O}$. From the magnetic hysteresis loop (M-H curve) of $\mathrm{Zn}_{0.96} \mathrm{Ni}_{0.04} \mathrm{O}$ (Fig.7), it is clear that the nanoparticles are ferromagnetic at room temperature. The values of their saturation magnetization $\left(M_{\mathrm{s}}\right)$ and coercive field $\left(H_{\mathrm{c}}\right)$ are $0.004 \mathrm{emu} / \mathrm{g}$ and $90 \mathrm{G}$.

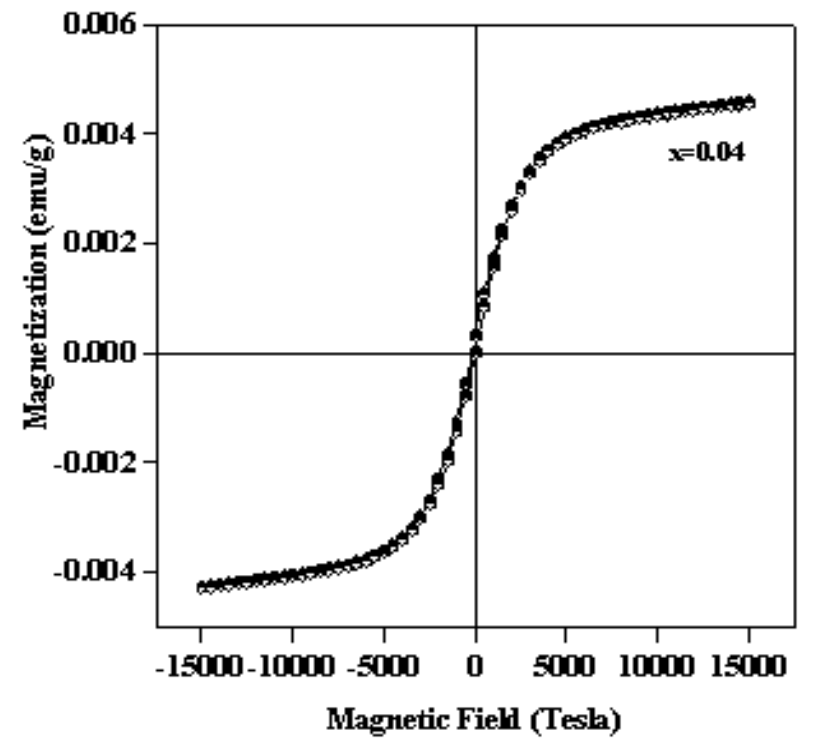

Figure 7. The magnetic hysteresis loops (M-H curve) of $\mathrm{Zn}_{0.96} \mathrm{Ni}_{0.04} \mathrm{O}$ sample

Though numerous work have been done on the transition metal doped $\mathrm{ZnO}$, the mechanism responsible for the origin of ferromagnetism at room temperature still remains controversial. So far, several assumptions have been made at the origin of ferromagnetism are: the role of the secondary phase, the connection between defects and magnetism, oxygen vacancy, etc.[16-18]. Among several models proposed to explain the origin of ferromagnetism in diluted magnetic semiconductor materials, carrier-mediated ferromagnetism explains the possible mechanism accountable for the magnetic behaviors. In our present $\mathrm{Zn}_{0.96} \mathrm{Ni}_{0.04} \mathrm{O}$ sample, the origin of ferromagnetism may be due to the formation of Ni-related secondary phase such as metallic nickel and NiO. But, from the structural study using XRD, NiO peaks are observed with the resolution limit of our XRD instrument. However, bulk $\mathrm{NiO}$ is antiferromagnetic with neel temperature of $520 \mathrm{~K}$ and hence the phase correlated to $\mathrm{NiO}$ can be ruled out. Thus, the room-temperature ferromagnetism in our $\mathrm{Zn}_{0.96} \mathrm{Ni}_{0.04} \mathrm{O}$ sample could originate from the long-range $\mathrm{Ni}^{2+}-\mathrm{Ni}^{2+}$ ferromagnetic coupling mediated by shallow donor electrons[19]. Thus, the origin of ferromagnetism in the sample could be due to the exchange interaction between free delocalized carriers and localized d spins on the nickel ions.

\subsection{Electron Paramagnetic Resonance}

EPR spectra of $\mathrm{Ni}$ (II) in $\mathrm{ZnO}$ have been recorded on a JEOL JES-TE100 ESR spectrometer operating at X-band frequency $9.38653 \mathrm{GHz}$ with microwave power of $1.00 \mathrm{~mW}$ in the field range $0-500 \mathrm{mT}$, having a $100 \mathrm{kHz}$ field modulation at $77 \mathrm{~K}$ to obtain the first derivative EPR spectrum. Fig. 8. shows the EPR spectra of nickel doped $\mathrm{ZnO}$ samples annealed at $800^{\circ} \mathrm{C}$. All spectra exhibit a symmetric and narrow resonance signal. From the EPR spectra of the samples, a minor shift of g-value from the free electron position being 2.002 is observed.

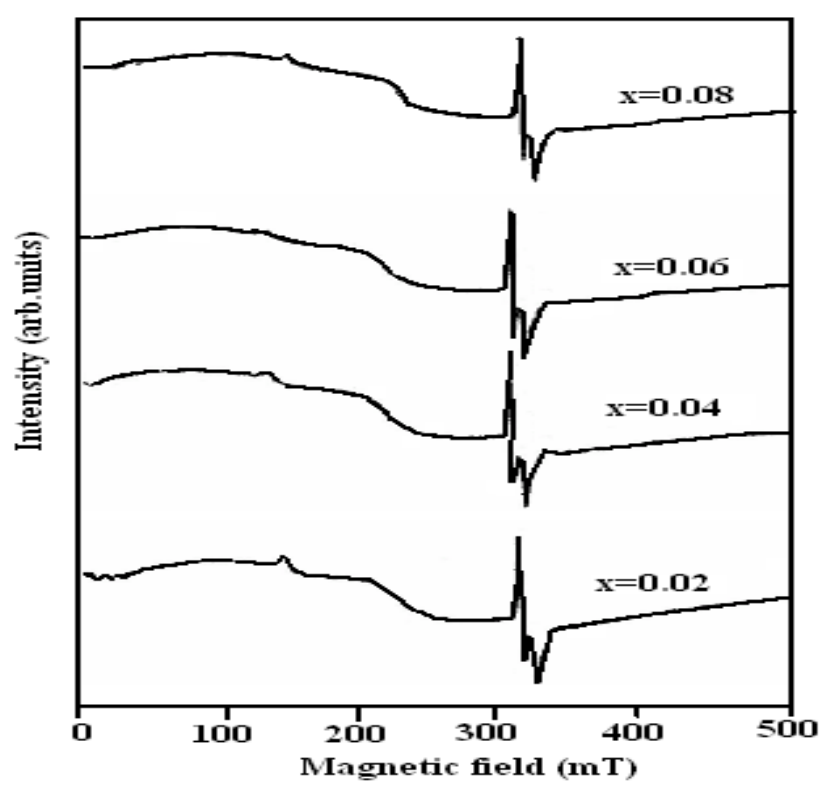

Figure 8. EPR spectrum of $\mathrm{Zn}_{1-\mathrm{x}} \mathrm{Ni}_{\mathrm{x}} \mathrm{O}(\mathrm{x}=0.02,0.04,0.06,0.08)$ $\mathrm{Ni}$-doped $\mathrm{ZnO}$ nanoparticles annealed at $800^{\circ} \mathrm{C}$

Clearly, it is observed from Fig. 8 that there is a slight decrease in the value of resonant field with the increase in $\mathrm{Ni}$ concentration. When the nickel content increases, the average distance between $\mathrm{Ni}^{2+}{ }_{-} \mathrm{Ni}^{2+}$ ions decreases and hence $\mathrm{Ni}$ atoms come close to each other. The super-exchange interactions between these neighboring $\mathrm{Ni}^{2+}$ ions increases the internal field[20] and decreases the resonant field with increasing nickel concentration.

\section{Conclusions}

In summary, nanocrystalline $\mathrm{Ni}$-doped $\mathrm{ZnO}$ were successfully synthesized by sol-gel auto-combustion method. $\mathrm{XRD}$ analysis reveals the formation of hexagonal wurtzite structure for all the nickel doped $\mathrm{ZnO}$ samples annealed at $800^{\circ} \mathrm{C}$. With increasing Ni content, phase segregation has 
occurred in the samples. Room temperature magnetic hysteresis loop of $\mathrm{Zn}_{1-\mathrm{x}} \mathrm{Ni}_{\mathrm{x}} \mathrm{O}(\mathrm{x}=0.04)$ sample confirms the ferromagnetic property of the sample. EPR analysis of the samples indicates that the resonant field decreases with increasing Ni content due to the super-exchange interactions between $\mathrm{Ni}^{2+}$ ions in the lattice.

\section{ACKNOWLEDGEMENTS}

The authors wish to thank the central instrumentation facility, Pondicherry University for providing TG/DTA, SEM, VSM and FTIR studies. We wish to thank UGC for funding XRD measurement at Department of physics, Pondicherry University, Puducherry and Department of Chemistry for providing EPR analysis.

\section{REFERENCES}

[1] Wolf, S. A., Awschalom D. D. and Buhrman, R. A., 2001, Science, 294, 1488.

[2] Pearton, S. J., Abernathy, C. R., Norton, D. P., Hebard, A. F., Park, Y. D., Boater L. A. and Budai, J. D., 2003, Mater. Sci. Eng., R 40, 137.

[3] Liu, E., Xiao, P., Chen, J. S., Lim, B. C. and Li, L., 2008, Current Applied Physics, 8, 408.

[4] Ohno, H, 1998, Science 281, 951.

[5] Chiba,, D., Takamura, K., Matsukura, F. and Ohno, H., 2003, Appl. Phys. Lett. 82, 3020.

[6] Dietl, T., Ohno, H., Matsukura, F., Cibert, J. and Ferrand, D., 2000, Science, 287, 1019.

[7] Xing, G., Wang, D., Yi, J., Yang, L., Gao, M. , He, M., Yang,
J., Ding, J., Sum T. and Wu, T., 2010, Appl. Phys. Lett. 96, 112511.

[8] El-Hilo, M., Dakhel A., and Ali-Mohamed, A., 2009, J. Magn. Magn. Mater. 321, 2279.

[9] Wang, H., Chen, Y., Wang, H. B., Zhang, C., Yang, F. J., Duan, J. X., Yang, C. P., Xu, Y. M., Zhou, M. J. and Li, Q., 2007 Appl. Phys. Lett. 90052505.

[10] Li, B. B., Xiu, X. Q., Zhang, R., Tao, Z. K., Chen, L., Xie, Z. L., Zheng, Y. D. and Xie, Z., 2006 Mater. Sci. Semicond. Proc. 9, 141.

[11] Cong, J., Hong, J. H., Liu, Q. Y., Liao, L. and Zhang, K. L., 2006, Solid State Commun. 138, 511.

[12] Ekambaram, S., 2005, J. Alloys Compd. 390, L4.

[13] Guangqing, P., Changtai, X., Shixun, C., Jungang, Z., Feng W. and Jun, X., 2006, J. Magn. Magn. Mater. 302, 340.

[14] Xingyu, M., Wei, Z. and Youwei, D., 2008, J. Magn. Magn. Mater. 320, 1102.

[15] Perales-Perez, O., Parra-Palomino, A., Singhal, R., Voyles, P. M., Zhu, Y., Jia, W. and Tomar, M. S., 2007, Nanotechnology, 18,315606 .

[16] Venkatesan, M., Fitzgerald, C. B. and Coey, J. M. D., 2004, Nature, 430, 630.

[17] Sluiter, M. H. F., Kawazoe, Y., Sharma, P., Inoue, A., Raju, A. R., Rout C., and Waghmare, U. V., 2005, Phys Rev Lett 94, 187204 .

[18] Eriksson, O., Bergqvist, L., Sanyal, B., Kudnovsky, J., Drchal, V., Korzhavyi P. and Turek, I., 2004, J. Phys. Condens. Matter 16, S5481.

[19] Coey, J. M. D., Venkatesan, M., and Fitzgerald, C. B., 2005 Nat. Mater. 4, 173.

[20] Liu, X. X., Lin, F. T., Sun, L. L., Cheng, W. J., Ma, X. M. and Shi, W. Z., 2006 Appl Phys Lett ,88, 062508. 